\title{
Non-Gaussian normal diffusion in a fluctuating corrugated channel
}

\author{
Yunyun Li, ${ }^{1}$ Fabio Marchesoni, ${ }^{1,2}$ Debajyoti Debnath, ${ }^{3}$ and Pulak K. Ghosh ${ }^{3, *}$ \\ ${ }^{1}$ Center for Phononics and Thermal Energy Science and Shanghai Key Laboratory of Special Microstructures, Materials and Technology, \\ School of Physics Science and Engineering, Tongji University, Shanghai 200092, People's Republic of China \\ ${ }^{2}$ Dipartimento di Fisica, Università di Camerino, I-62032 Camerino, Italy \\ ${ }^{3}$ Department of Chemistry, Presidency University, Kolkata 700073, India
}

(Received 7 May 2019; published 1 October 2019)

\begin{abstract}
A Brownian particle floating in a narrow corrugated (sinusoidal) channel with fluctuating cross section exhibits non-Gaussian normal diffusion. Its displacements are distributed according to a Gaussian law for very short and asymptotically large observation times, whereas a robust exponential distribution emerges for intermediate observation times of the order of the channel fluctuation correlation time. For intermediate to large observation times, the particle undergoes normal diffusion with one and the same effective diffusion constant. These results are analytically interpreted without having recourse to heuristic assumptions. Such a simple model thus reproduces recent experimental and numerical observations obtained by investigating complex biophysical systems.
\end{abstract}

DOI: 10.1103/PhysRevResearch.1.033003

\section{INTRODUCTION}

Recent observations [1-6] of particle diffusion in fluctuating crowded environments manifestly contradict the common belief that normal diffusion is associated with a Gaussian distribution of spatial displacements. Indeed, if for simplicity, we restrict ourselves to one dimensional (1D) geometries, the displacement, $\Delta x(t)=x(t)-x(0)$, of a standard overdamped Brownian particle suspended in a homogeneous Newtonian fluid [7] (i) grows with time according to the EinsteinStokes law, $\left\langle\Delta x^{2}(t)\right\rangle=2 D t$; and (ii) is distributed according to a rescaled Gaussian probability density function (pdf), $p(\Delta x / \sqrt{t})$ with half-variance $D$. Under these circumstances, the random variable $\Delta x(t)$ is said to undergo Gaussian normal (or Fickian) diffusion.

There is no a priori reason why the diffusion of a tracer in a time varying inhomogeneous medium should be Fickian. In real biophysical systems, with increasing the observation time the rescaled displacement distributions, $p(\Delta x / \sqrt{t})$, often develop prominent exponential tails, whereas the tracers start diffusing linearly in time. Such transient tails disappear only for asymptotically large observation times (at times hardly accessible to real experiments [1]), when finally the $\Delta x$ distributions turn Gaussian, as predicted by the central limit theorem, without appreciably changing the underlying diffusion mechanism. Persistent diffusive transients of this type have been detected in diverse experimental setups [1-3,8-10]. Extensive numerical simulations confirmed the occurrence

\footnotetext{
*Corresponding author: pulak.chem@ presiuniv.ac.in

Published by the American Physical Society under the terms of the Creative Commons Attribution 4.0 International license. Further distribution of this work must maintain attribution to the author(s) and the published article's title, journal citation, and DOI.
}

of this remarkable phenomenon in crowded environments consisting of slowly diffusing or changing microscopic constituents (filaments [1,3], large hard spheres [4-6], clusters $[11,12]$, and other heterogeneities [13]).

The current interpretation of picture above postulates the existence of one or more relaxation processes affecting the suspension medium or the confining geometry, where the tagged particle diffuses [1]. As long as the fixed time interval, $t$, over which $\Delta x(t)$ is measured is of the order of the relaxation time constant(s), $\tau$, the particle displacement can obey a non-Gaussian statistics. Through what mechanism, under these conditions the particle's diffusion retains its normal character, may vary from case to case. To this purpose, a popular paradigm revolves around the heuristic notion of diffusing diffusivity [14], whereas the environmental fluctuations are modeled by means of an ad hoc random particle diffusion constant, $D(t)$. On assuming that $D(t)$ is an Ornstein-Ulenbeck process with average $D$ and time constant $\tau$, the distribution $p(\Delta x / \sqrt{t})$ changes from exponential for $t \ll \tau$ to Gaussian for $t \gg \tau$. In both time regimes, the displacement diffusion is normal, with $\left\langle\Delta x^{2}(t)\right\rangle=2 D t$ [14]. This phenomenological description, together with its more refined variations [15-21], may qualitatively interpret a conspicuous body of diverse experimental observations, but sheds little light on the underlying microscopic mechanisms.

In this paper, we investigate both numerically and analytically the directed diffusion of an overdamped Brownian particle in a narrow quasi-1D corrugated channel [22,23] of fluctuating width. Such a time variable geometry is inspired to cell biology [24,25] and models the key ingredient of the phenomenon under study, namely slow environmental fluctuations. The relevant stochastic model is detailed in Sec. II. The simulation results of Sec. III reproduce the essentials of non-Gaussian normal diffusion [14] without having recourse to the paradigm of diffusing diffusion: (1) the distribution of the particle displacements along the channel is Gaussian 
for observation times either much shorter (local diffusion) or much longer than the correlation time of the channel fluctuation (channel diffusion), and exponential for a rather wide interval of intermediate observation times. (2) A normal diffusion law with the same channel diffusion constant extends from intermediate to large observation times, thus implying a nontrivial relationship between displacement pdf's. The compatibility of normal diffusion with different displacement statistics is discussed in Sec. IV. (3) These effects are robust as long as fluctuations randomly open and close the channel constrictions. As remarked in the concluding Sec. V, the compartmentalization of particle's diffusion thus emerges as a prerequisite of non-Gaussian normal diffusion.

\section{FLUCTUATING CHANNEL MODEL}

The dynamics of an overdamped (or massless) Brownian particle in a channel is modeled by the Langevin equation $\dot{\mathbf{r}}(t)=\sqrt{D_{0}} \boldsymbol{\xi}(t)$, where $\mathbf{r}=(x, y)$ are the particle's coordinates and the translational fluctuations $\boldsymbol{\xi}(t)=\left[\xi_{x}(t), \xi_{y}(t)\right]$ are zero-mean, white Gaussian noises with autocorrelation functions $\left\langle\xi_{i}(t) \xi_{j}(0)\right\rangle=2 \delta_{i, j} \delta(t)$, with $i, j=x, y$. The strength of $\xi_{i}(t)$ is the free-particle diffusion constant, $D_{0}$, which is typically proportional to the temperature of the suspension fluid. Without loss of generality, we considered a twodimensional (2D) sinusoidal channel with axis oriented along $x$ and symmetrically confined transverse coordinate, $|y| \leqslant$ $w(x, t)$, where

$$
w(x, t)=\left(y_{L} / 2\right)\left[\varepsilon^{2}+\left(1-\varepsilon^{2}\right) \sin ^{2}\left(\pi x / x_{L}\right)\right] .
$$

Here, $y_{L}$ and $x_{L}$ are respectively the maximum width and the length of the unit channel cell, and $\varepsilon^{2} y_{L}$ is the fluctuating width of the pores located at $x=0 \bmod (\pi)$. We assume for simplicity that $\varepsilon(t)$ obeys the Ornstein-Uhlenbeck equation

$$
\dot{\varepsilon}=-\left(\varepsilon-\varepsilon_{0}\right) / \tau+\sqrt{D_{\varepsilon} / \tau^{2}} \xi_{\varepsilon}(t),
$$

where the noise $\xi_{\varepsilon}(t)$ has the same statistics of, but is uncorrelated with the thermal noises, $\boldsymbol{\xi}(t)$. Unless stated otherwise, we set $\varepsilon_{0}=0$, so that the average pore width is $\left\langle\varepsilon^{2}\right\rangle y_{L}$ with $\left\langle\varepsilon^{2}\right\rangle \doteq \sigma_{\varepsilon}^{2}=D_{\varepsilon} / \tau$. In order to ignore hydrodynamic effects [23], we addressed the case of pointlike particles in highly viscous suspension fluids. Accordingly. we assumed that, for small channel fluctuations, the varying pressure exerted by the walls on the fluid does not sensibly modulate the particle's diffusion constant, $D_{0}$, neither in space nor in time. In practice, to modulate the effective width of the channel pores without incurring this difficulty, one can simply apply a tunable external gating potential [23].

We numerically integrated the particle Langevin equation in the free space inside the channel by means of a Milstein algorithm [26]; we imposed reflecting boundary conditions at the channel's walls, $y= \pm w(x, t)$, and took stochastic averages over not fewer than $10^{5}$ particle's trajectories with random initial conditions.

\section{THE CASE OF OPENING-CLOSING PORES}

The statistics of the particle displacement, $\Delta x(t)$, depends on the observation time, $t$, as shown in Fig. 1, where three different pdf regimes are clearly

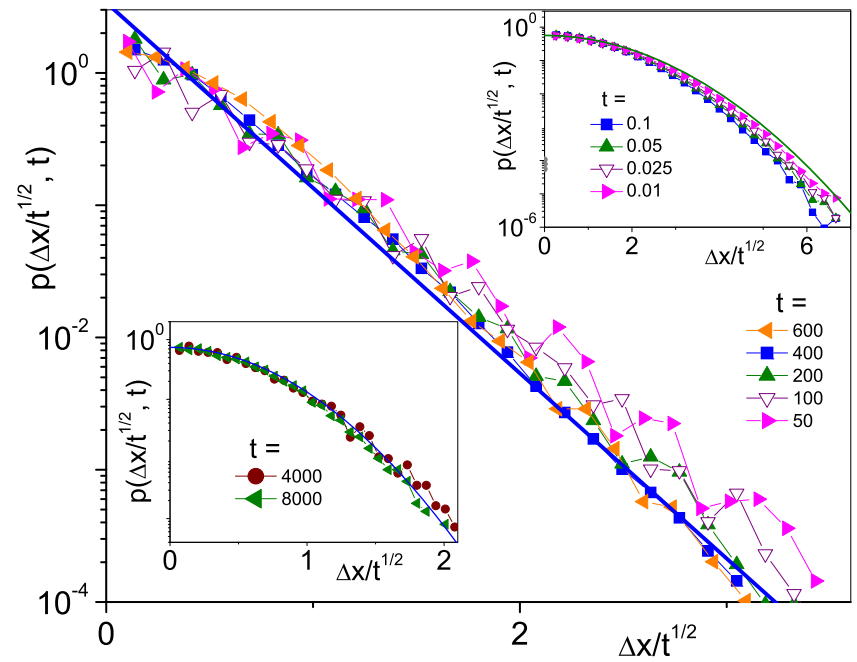

FIG. 1. Diffusion regimes in a fluctuating channel. The rescaled displacement pdf's, $p(\Delta x / \sqrt{t})$, are computed for short, large, and intermediate observation times $t$, respectively, in the top-right inset, bottom-left inset, and main panel. Simulation parameters are: $y_{L}=1, x_{L}=\pi, D_{0}=1, D_{\varepsilon}=3, \varepsilon_{0}=0$, and $\tau=200$. The relevant Gaussian and Laplace distributions are represented by solid curves. The half-variance of the Gaussian fitting curves in the insets are $B=$ $D_{0}=1$ (top right) and $B=D=0.145$ (bottom left); the exponential decay constant is $\alpha=0.325$ (main panel). The parameters $\alpha$ and $B$ are defined in the text.

distinguishable: two distinct Gaussian distributions, $p(\Delta x / \sqrt{t})=(4 \pi B)^{-1 / 2} \exp \left(-\Delta x^{2} / 4 B t\right)$, at very short and large $t$ values (insets) and an exponential (or Laplace) distribution, $p(\Delta x / \sqrt{t})=(2 \alpha)^{-1} \exp (-\Delta x / \alpha \sqrt{t})$, over an extended intermediate $t$ range. The short- $t$ Gaussian regime describes the free Brownian diffusion inside a single channel cell, far from the walls, which occurs for time intervals not larger than $\tau_{L}=\min \left\{x_{L}^{2} / 8 D_{0}, y_{L}^{2} / 8 D_{0}\right\}$. Under these circumstances, the fitting parameter $B$ turned out to coincide with $D_{0}$, as expected [7].

For larger observation times, the particle becomes sensitive to confinement [22]. The escape from one cell into the adjacent ones requires diffusing through narrow pores, a mechanism that takes relatively long waiting times. On extending the approximate techniques of Ref. [27] to the case of fluctuating pores, one estimates a characteristic mean-first exit time (MFET)

$$
\tau_{0}=\frac{x_{L}^{2}}{8 D_{0}} \frac{1}{\langle|\varepsilon|\rangle}=\frac{x_{L}^{2}}{8 D_{0}} \sqrt{\frac{\pi \tau}{2 D_{\varepsilon}}} .
$$

Here, $\tau_{0}$ is the time the particle takes to diffuse from inside a cell up to the center of its left or right exit pore. Accordingly, the time constant of the corresponding discrete intercell jumping process is $2 \tau_{0}$ and the channel diffusion constant is thus well approximated by $[23,27]$

$$
D=x_{L}^{2} / 4 \tau_{0} \text {. }
$$

On the other hand, the correlation time of the fluctuating pore width, $\varepsilon^{2}(t)$, is $2 \tau$, see Eq. (2). In view of these timescales, one is led to anticipate that the three distinct regimes of the 


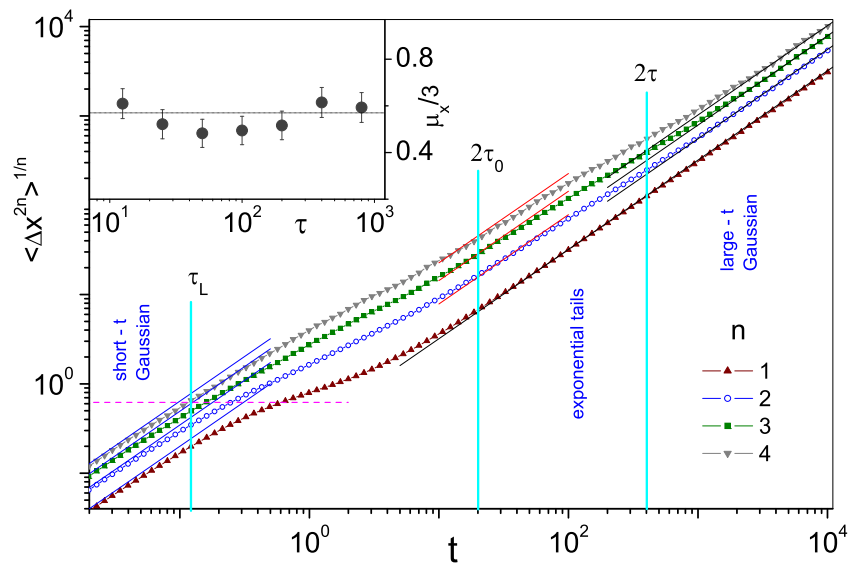

FIG. 2. Normal diffusion in a fluctuating channel: time dependence of $\left\langle\Delta x^{2 n}\right\rangle^{1 / n}$ for different $n$. Simulation parameters are: $y_{L}=1$, $x_{L}=\pi, D_{0}=1, \varepsilon_{0}=0, D_{\varepsilon}=3$, and $\tau=200$. The parallel straight lines represent the expected values for the higher order moments $(n>1)$ in terms of the fitted normal diffusion law for $\left\langle\Delta x^{2}(t)\right\rangle$ in the Gaussian and exponential regimes (see text). The vertical lines delimit the three pdf regimes of Fig. 1 and are positioned respectively at $t=\tau_{L}, 2 \tau_{0}$ and $2 \tau$. (Inset:) Ratio $\mu_{x} / 3 \simeq \alpha^{2} / B$ vs $\tau$ at large $t$. The numerical quantifier $\mu_{x}$ is defined in Sec. IV and was computed here for $t=2 \tau$; for an ideal exponential transient $\mu_{x} / 3=\pi / 2-1$ (horizontal line).

rescaled pdf illustrated in Fig. 1 must hold for observation times $t \ll \tau_{L}, 2 \tau_{0} \lesssim t \lesssim 2 \tau$ and $t \gg 2 \tau$, respectively.

In the asymptotic regime, $t \gg 2 \tau$, the particle diffusion along the channel is normal (i.e., $\left\langle\Delta x^{2}\right\rangle$ is a linear function of $t$, Fig. 2), which allows a direct numerical determination of the constant $D$ of Eq. (4). Moreover, the intercell jumping process yields a Gaussian distribution of the discretized particle displacements (Brownian random walker [7]). This suggests that asymptotically $B=D$, as discussed below.

The exponential transient, $2 \tau_{0} \lesssim t \lesssim 2 \tau$, thus bridges two Gaussian limits, $t \rightarrow 0$ with $B=D_{0}$, and $t \rightarrow \infty$ with $B=$ $D$. Most remarkably, the same Laplace law (i.e., one decay constant $\alpha$ ) fits all $\Delta x / \sqrt{t}$ distributions over about one decade of observation times. The similarity with recent experimental observations is apparent [1-6]. The short- $t$ Gaussian regime cannot be reproduced by the diffusing diffusion model of Ref. [14], as there the intracell diffusion time, $\tau_{L}$, was implicitly set to zero. We notice that the exponential transient interval can be expanded by decreasing $\sigma_{\epsilon}^{2}$ while increasing $\tau$. In the present case, however, this condition leads soon to extremely long simulation runs.

A defining property of channel diffusion is featured in Fig. 2. One normal diffusion law with the same diffusion constant, $D$, fits all numerical data for $\left\langle\Delta x^{2}(t)\right\rangle$ with $t \gtrsim$ $2 \tau_{0}$, i.e., in correspondence with both the Laplace and the asymptotic Gaussian distributions. To assess the Gaussian nature of the normal diffusion for $t \rightarrow 0$ and $t \rightarrow \infty$, we explicitly computed a few higher moments $\left\langle\Delta x^{2 n}\right\rangle$ with $n \geqslant$ 1 , also reported in Fig. 2. We checked that in both limits $\left\langle\Delta x^{2 n}\right\rangle^{1 / n}=[(2 n-1) ! !]^{1 / n}\left\langle\Delta x^{2}\right\rangle$, as expected for Gaussian $\Delta x$ distributions. Instead, for the Laplace pdf's fitted in Fig. 1, one would expect $\left\langle\Delta x^{2 n}\right\rangle^{1 / n}=\left[(2 n !) / 2^{n}\right]^{1 / n}\left\langle\Delta x^{2}\right\rangle$. The agreement between these latter estimates and the actual
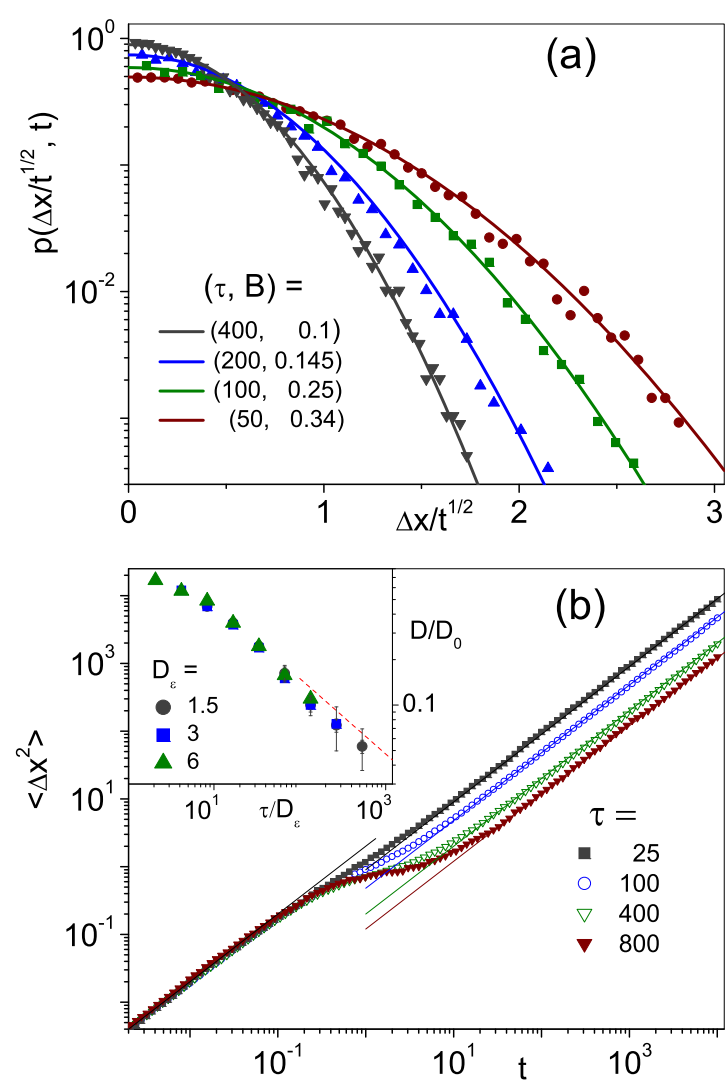

FIG. 3. Role of the fluctuation time correlation: (a) displacement pdf, $p(\Delta x / \sqrt{t})$, and (b) diffusion, $\left\langle\Delta x^{2}\right\rangle$ vs $t$ for increasing $\tau$. Simulation parameters are: $y_{L}=1, x_{L}=\pi, D_{0}=1, \varepsilon_{0}=0$, and $D_{\varepsilon}=3$. The straight lines on the right-hand side of (b) are the linear fits employed to extract the $D$ constant, whereas the solid lines in (a) are the corresponding Gaussian curves, $p(\Delta x / \sqrt{t})$, defined in the text, with $B=D$. For short $t$, all curves in (b) collapse on one linear branch with diffusion constant $D_{0}$, also denoted by a straight line. (Inset) $D$ vs $\tau$ for different $D_{\varepsilon}$ (see legend). All other simulation parameters are as in the main panel.

diffusion data in the intermediate $t$ domain is qualitative good, only, which we attributed to the deviations from the Laplace distributions, apparent at $\Delta x / \sqrt{t} \ll \alpha$.

On the other hand, the Laplace and the large- $t$ Gaussian fitting pdf curves introduced above, yield the same channel diffusion constant, $D$, only under the condition $\alpha^{2}=B$. However, the inset of Fig. 2 shows that, for the parameters of Fig. $1,0.8<\alpha / \sqrt{B}<0.9$. The ratio $\alpha / \sqrt{B}$ thus serves as a measure of the exponential character of the displacement statistics across the normal diffusion transient dominated by channel fluctuations. An estimate of this ratio is obtained in Sec. IV.

The role of the timescale $\tau$ is further illustrated in Fig. 3 . As the MFET of Eq. (3) increases like $\tau^{1 / 2}$, for large $\tau$ the diffusion curves $\left\langle\Delta x^{2}(t)\right\rangle$ develop a plateau in the range $\tau_{L} \ll t \ll \tau_{0}$. During this time interval, the diffusing particle "fills up" the 2D channel cell where it was initially injected, attaining a temporary maximum displacement $\left\langle\Delta x^{2}\right\rangle \simeq \bar{x}^{2}$, where $\bar{x}=\pi / 4$ is the average half-width of the cell $w(x)$, Eq. (1), for $\sigma_{\varepsilon} \rightarrow 0$. This diffusion plateau is represented in Fig. 2 by a horizontal line. 
The numerical estimates of the channel diffusion constant, $D$, for $t \gtrsim 2 \tau_{0}$ and the fitting parameter $B$ of the Gaussian $\Delta x$ pdf for $t \gg 2 \tau$ were anticipated to coincide. Simulation data. like those reported in Fig. 3, confirmed our expectations, within the statistical error, for all choices of the simulation parameters. Moreover, on combining Eqs. (3) and (4), one expects that $D / D_{0}=2\left(2 D_{\varepsilon} / \pi \tau\right)^{1 / 2}$, also in fairly close agreement with the numerical data plotted in the inset of Fig. 3(b).

Finally, we notice that on lowering $\tau$ the slopes of the normal diffusion branches for $t \ll \tau_{L}$ and $t \gtrsim 2 \tau_{0}$ tend to coincide, that is $D$ (and $B$ ) tend to $D_{0}$. This is due to the fact that the average pore width, $\left\langle\varepsilon^{2}\right\rangle y_{L}$, grows like $D_{\varepsilon} / \tau$. As a result, for $\langle\varepsilon\rangle \geqslant 1$ the effective channel bottlenecks are no longer located at $x=0 \bmod \{\pi\}$, but rather at $x=\pi / 2 \bmod \{\pi\}$, and have fixed width, $y_{L}$. Accordingly, from Eq. (3), $\tau_{0}=x_{L}^{2} / 8 D_{0}$ and the channel diffusion constant of Eq. (4) is $D=D_{0}$.

\section{EXPONENTIAL NORMAL DIFFUSION}

In Sec. III, we showed that on increasing the observation time larger than twice the MFET, (i) the displacement distribution evolves from Laplacian to Gaussian, and (ii) the same normal diffusion law holds for any $t$. A simple argument, first introduced in Ref. [14], can be generalized to support these conclusions.

Let us model a particle trajectory in the $x$ direction as the sum of random small steps, $\Delta x_{i}$, taken at discretized times, $t_{i}=i \Delta t$, where $i=1, \ldots N$, and $\Delta t=1$, for simplicity. The position of the particle at time $N$ is, therefore, $x_{N}=\sum_{i=1}^{N} \Delta x_{i}$. Accordingly,

$$
\left\langle x_{N}^{2}\right\rangle=\sum_{i=1}^{N}\left\langle\Delta x_{i}^{2}\right\rangle+2 \sum_{i \neq j}{ }^{\prime}\left\langle\Delta x_{i} \Delta x_{j}\right\rangle,
$$

where $\sum_{i \neq j}^{\prime}$ stays for $\sum_{i=1}^{N-1} \sum_{j=1+1}^{N}$. Contrary to the standard model of Brownian random walker [7], normal diffusion at time $N$ sets in under the generic condition that the step directions are uncorrelated, $\left\langle\Delta x_{i} \Delta x_{j}\right\rangle=0$, that is for mirrorsymmetric distributions, $p\left(\Delta x_{i}\right)$, with variances, $\left\langle\Delta x_{i}^{2}\right\rangle$, possibly different, but of the same order.

Following the authors of Ref. [14], one can further assume that during each unit time step the particle's diffusion is normal with time-dependent constant, $D_{i}$, i.e.,

$$
p\left(\Delta x_{i}\right)=\left(4 \pi D_{i}\right)^{-1 / 2} \exp \left(-\Delta x_{i}^{2} / 4 D_{i}\right),
$$

with unspecified $D_{i}$ distribution, $p\left(D_{i}\right)$. It follows immediately that

$$
\left\langle x_{N}^{2}\right\rangle=2\langle D\rangle N
$$

and

$$
\begin{aligned}
\left\langle x_{N}^{4}\right\rangle-3\left\langle x_{N}^{2}\right\rangle^{2}= & 12\left(\left\langle D^{2}\right\rangle-\langle D\rangle^{2}\right) N \\
& +24 \sum_{i \neq j}^{\prime}\left(\left\langle D_{i} D_{j}\right\rangle-\left\langle D_{i}\right\rangle\left\langle D_{j}\right\rangle\right),
\end{aligned}
$$

where $\langle D\rangle \equiv\left\langle D_{i}\right\rangle$ for the relevant choice of $p\left(D_{i}\right)$.

Suppose now that two particle steps, $\Delta x_{i}$ and $\Delta x_{j}$ are statistically uncorrelated, i.e., $\left\langle D_{i} D_{j}\right\rangle=\left\langle D_{i}\right\rangle\left\langle D_{j}\right\rangle$, only for $|i-j|>\tau$. We then distinguish two limiting cases: (i) $N \gg \tau$, where

$$
\mu_{x}=\frac{\left\langle x_{N}^{4}\right\rangle-3\left\langle x_{N}^{2}\right\rangle^{2}}{\left\langle x_{N}^{2}\right\rangle^{2}}=\frac{3 \mu_{D}}{N} \rightarrow 0,
$$

with $\mu_{D}=\left(\left\langle D^{2}\right\rangle-\langle D\rangle^{2}\right) /\langle D\rangle^{2}$. A vanishing $\mu_{\mathrm{x}}$ hints at a Gaussian $x_{N}$ distribution as obtained from numerical simulation.

(ii) $N \ll \tau$, where

$$
\mu_{x}=\frac{\left\langle x_{N}^{4}\right\rangle-3\left\langle x_{N}^{2}\right\rangle^{2}}{\left\langle x_{N}^{2}\right\rangle^{2}} \simeq 3 \mu_{D} .
$$

$\mu_{\mathrm{x}}=3$ would correspond to an exponential distribution of $x_{N}$; the coefficient $\mu_{D}$ is thus a measure of the deviation of the actual $x_{N}$ distribution from the ideal Laplace distribution.

To apply the argument above to the model under study, the time step $\Delta t$ has to be taken not shorter than $\tau_{0}$, i.e., the argument does not hold for the intracell diffusion. The corresponding coefficient $\mu_{D}$ can be estimated analytically by adapting the procedure of Ref. [27] to the case of a fluctuating channel, namely,

$$
\mu_{D} \simeq \frac{\left\langle\varepsilon^{2}\right\rangle-\langle\varepsilon\rangle^{2}}{\langle\varepsilon\rangle^{2}}=\frac{\pi}{2}-1 .
$$

On the other hand, on adopting the Laplace distribution $p(\Delta x / \sqrt{t})$ for $x_{N}$ and the normal diffusion law, $\left\langle\Delta x^{2}\right\rangle=$ $2 D t$, instead of Eq. (6), that is, $\langle D\rangle=D=B$, numerator and denominator of $\mu_{\mathrm{x}}$ can be calculated explicitly to obtain

$$
\mu_{x}=\frac{3 \alpha^{4}}{B^{2}} .
$$

Finally, on comparing Eqs. (9)-(11), one estimates $\alpha / B^{1 / 2} \simeq$ 0.86 , in fairly close agreement with the numerical data reported in the inset of Fig. 2.

\section{CONCLUSIONS}

So far, by setting $\varepsilon_{0}=0$ in Eq. (2) we assumed that the fluctuations of the channel cause the opening and closing of its pores. Of course, in most cases the pores remain open at all times, with $\varepsilon_{0}>0$ and slightly fluctuating cross-section. Therefore we investigated how the non-Gaussian normal diffusion mechanism depends on $\varepsilon_{0}$. In the main frame of Fig. 4, we plotted the rescaled displacement pdf's at $t=2 \tau$ for the simulation parameters of Fig. 1, except that $\varepsilon_{0}$ is increased from 0 up to well above $\sigma_{\varepsilon}$. One sees immediately that on widening the pores, the rescaled pdf's change from exponential at $\varepsilon_{0}=0$, see Fig. 1 , to Gaussian with $B=D$, for $\varepsilon_{0} \gtrsim \sigma_{\varepsilon}$. The dependence of $D$ on $\varepsilon_{0}$ in the absence and presence of channel fluctuations is compared in the inset of Fig. 4. For $\varepsilon_{0} \gtrsim \sigma_{\varepsilon}$, the diffusion constant grows insensitive to the fluctuation strength, $D_{\varepsilon}$. The obvious conclusion is that non-Gaussian normal diffusion only occurs when the fluctuations of the channel walls are strong enough to actually open and close the pores. Note that in the absence of channel fluctuations, $D_{\varepsilon}=0$, our data for $D$ are well fitted by Eq. (4), with $\tau_{0}$ given by the first Eq. (3) upon replacing $\langle\varepsilon\rangle$ with $\varepsilon_{0}[27]$. 


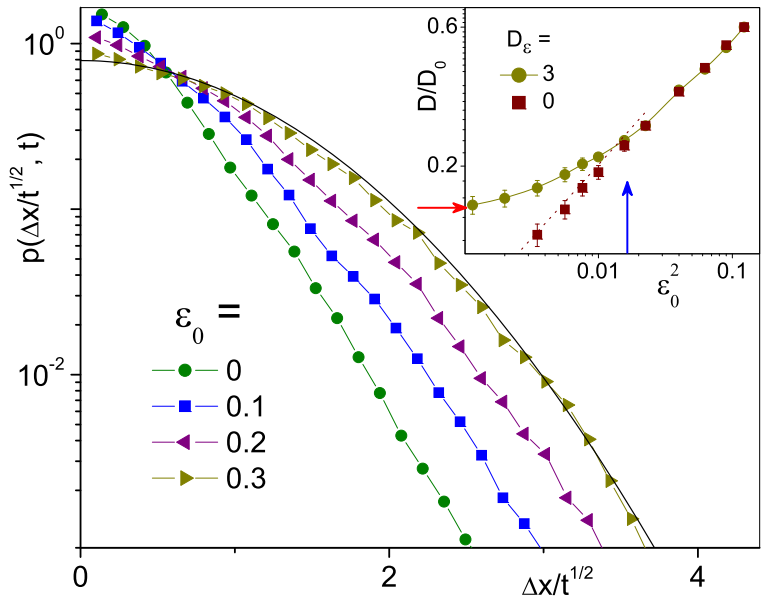

FIG. 4. Role of the fixed pore width $\varepsilon_{0}: p(\Delta x / \sqrt{t})$ at $t=2 \tau$ for increasing $\varepsilon_{0}$ (see legend). Other simulation parameters are: $y_{L}=1$, $x_{L}=\pi, D_{0}=1, D_{\varepsilon}=3$, and $\tau=200$. The solid curve represents a normalized Gaussian distribution with $B=D, D$ being fitted from the normal diffusion data (inset). (Inset) Channel diffusion constant, $D$, as a function of $\varepsilon_{0}$ in the presence $\left(D_{\varepsilon}=3\right)$ and absence $\left(D_{\varepsilon}=0\right)$ of channel fluctuations. The dashed line is the analytical prediction $D / D_{0}=2 \varepsilon_{0}$ [27]; the values $\varepsilon_{0}^{2}=\sigma_{\varepsilon}^{2}$ and $D / D_{0}$ at $\varepsilon_{0}=0$ are denoted by a vertical and a horizontal arrow, respectively.

The results of Fig. 4 illustrate the importance of diffusion compartmentalization during intermediate observation time intervals, $2 \tau_{0} \lesssim t \lesssim 2 \tau$. For $\varepsilon_{0}>\sigma_{\varepsilon}$, the tagged particle diffuses along the channel at all times, with only weakly correlated open-pore crossings; hence a Gaussian displacement distribution. In sharp contrast, for $\varepsilon_{0}<\sigma_{\varepsilon}$, the pore crossings of the trapped particle grow more and more time correlated; hence the exponential tails of $p(\Delta x / \sqrt{t})$ discussed in Sec. IV. This description is consistent with the subordination mechanism advocated in Ref. [20].

The microscopic model investigated in this paper, despite its simplicity, was proven to reproduce most of the intriguing properties of the phenomenon known as non-Gaussian normal diffusion. Such a phenomenon has emerged as ubiquitous in soft matter physics, which suggests a number of promising generalizations of the present model by incorporating additional sources of randomness [28], for instance, by decorrelating channel's pore spacing and fluctuations [29] or exciting size and configurational fluctuations of the diffusing particles [30], each on a suitably long timescale. Their combined action is expected to make the conclusions of the present study even more robust.

\section{ACKNOWLEDGMENTS}

Y.L. is supported by the NSF China under Grant No. 11875201. P.K.G. is supported by SERB Start-up Research Grant (Young Scientist) No. YSS/2014/000853 and the UGC-BSR Start-Up Grant No. F.30-92/2015. D.D. thanks Council of Scientific and Industrial Research (CSIR), Government of India, for support through a Junior Research Fellowship.
[1] B. Wang, S. M. Anthony, S. C. Bae, and S. Granick, Anomalous yet Brownian, Proc. Natl. Acad. Sci. USA 106, 15160 (2009).

[2] B. Wang, J. Kuo, C. Bae, and S. Granick, When Brownian diffusion is not Gaussian, Nat. Mater. 11, 481 (2012).

[3] S. Bhattacharya, D. K. Sharma, S. Saurabh, S. De, A. Sain, A. Nandi, and A. Chowdhury, Plasticization of poly(vinylpyrrolidone) thin films under ambient humidity: Insight from single-molecule tracer diffusion dynamics, J. Phys. Chem. B 117, 7771 (2013).

[4] J. Kim, C. Kim, and B. J. Sung, Simulation Study of Seemingly Fickian but Heterogeneous Dynamics of Two Dimensional Colloids, Phys. Rev. Lett. 110, 047801 (2013).

[5] G. Kwon, B. J. Sung, and A. Yethiraj, Dynamics in crowded environments: Is non-Gaussian Brownian diffusion normal? J. Phys. Chem. B 118, 8128 (2014).

[6] J. Guan, B. Wang, and S. Granick, Even hard-sphere colloidal suspensions display Fickian yet non-Gaussian diffusion, ACS Nano 8, 3331 (2014).

[7] C. Gardiner, Stochastic Methods: A Handbook for the Natural and Social Sciences (Springer, Berlin, 2009)

[8] E. R. Weeks, J. C. Crocker, A. C. Levitt, A. Schofield, D. A. Weitz, Three-dimensional direct imaging of structural relaxation near the colloidal glass transition, Science 287, 627 (2000).
[9] J. D. Eaves and D. R. Reichman, Spatial dimension and the dynamics of supercooled liquids, Proc. Natl. Acad. Sci. USA 106, 15171 (2009).

[10] K. C. Leptos, J. S. Guasto, J. P. Gollub, A. I. Pesci, and R. E. Goldstein, Dynamics of Enhanced Tracer Diffusion in Suspensions of Swimming Eukaryotic Microorganisms, Phys. Rev. Lett. 103, 198103 (2009).

[11] W. K. Kegel and A.van Blaaderen, Direct observation of dynamical heterogeneities in colloidal hard-sphere suspensions, Science 287, 290 (2000).

[12] P. Chaudhuri, L. Berthier, and W. Kob, Universal Nature of Particle Displacements Close to Glass and Jamming Transitions, Phys. Rev. Lett. 99, 060604 (2007).

[13] W. He, H. Song, Y. Su, L. Geng, B. J. Ackerson, H. B. Peng, and P. Tong, Dynamic heterogeneity and non-Gaussian statistics for acetylcholine receptors on live cell membrane, Nat. Commun. 7, 11701 (2016).

[14] M. V. Chubynsky and G. W. Slater, Diffusing Diffusivity: A Model for Anomalous, Yet Brownian, Diffusion, Phys. Rev. Lett. 113, 098302 (2014).

[15] A. G. Cherstvy and R. Metzler, Anomalous diffusion in timefluctuating non-stationary diffusivity landscapes, Phys. Chem. Chem. Phys. 18, 23840 (2016).

[16] R. Jain and K. L. Sebastian, Diffusion in a crowded, rearranging environment, J. Phys. Chem. B 120, 3988 (2016). 
[17] R. Jain and K. L. Sebastian, Diffusing diffusivity: A new derivation and comparison with simulations, J. Chem. Sci. 129, 929 (2017).

[18] N. Tyagi and B. J. Cherayil, Non-Gaussian Brownian diffusion in dynamically disordered thermal environments, J. Phys. Chem. B 121, 7204 (2017).

[19] L. Luo and M. Yi, Non-Gaussian diffusion in static disordered media, Phys. Rev. E 97, 042122 (2018).

[20] A. V. Chechkin, F. Seno, R. Metzler, and I. M. Sokolov, Brownian Yet Non-Gaussian Diffusion: From Superstatistics to Subordination of Diffusing Diffusivities, Phys. Rev. X 7, 021002 (2017).

[21] J. Slezak, R. Metzler, and M. Magdziarz, Superstatistical generalised Langevin equation: non-Gaussian viscoelastic anomalous diffusion, New J. Phys. 20, 023026 (2018).

[22] P. S. Burada, P. Hänggi, F. Marchesoni, G. Schmid, and P. Talkner, Diffusion in confined geometries, Chem. Phys. Chem. 10, 45 (2009).

[23] X. Yang, C. Liu, Y. Li, F. Marchesoni, P. Hänggi, and H. P. Zhang, Hydrodynamic and entropic effects on colloidal diffusion in corrugated channels, Proc. Natl. Acad. Sci. USA 114, 9564 (2017).

[24] P. Hänggi and F. Marchesoni, Artificial brownian motors: Controlling transport on the nanoscale, Rev. Mod. Phys. 81, 387 (2009).

[25] R. Lipowsky, Generic interactions of flexible membranes, in Handbook of Biological Physics, edited by R. Lipowsky and E. Sackmann (Elsevier, Amsterdam, 1995), Vol. 1, Chap. 11.

[26] P. E. Kloeden and E. Platen, Numerical Solution of Stochastic Differential Equations (Springer, Berlin, Heidelberg, 1992).

[27] L. Bosi, P. K. Ghosh, and F. Marchesoni, Analytical estimates of free Brownian diffusion times in corrugated narrow channels, J. Chem. Phys. 137, 174110 (2012).

[28] S. Marbach, D. S. Dean and L. Bocquet, Transport and dispersion across wiggling nanopores, Nat. Phys. 14, 1108 (2018).

[29] J. Wang, Y. Zhang, and H. Zhao, Non-Gaussian normal diffusion induced by delocalization, Phys. Rev. E 93, 032144 (2016).

[30] M. A. Cohen Stuart et al., Emerging applications of stimuliresponsive polymer materials, Nat. Mater. 9, 101 (2010). 